\title{
Collective enhancement and suppression in Bose-Einstein condensates
}

\author{
Wolfgang Ketterle, Ananth P. Chikkatur, and Chandra Raman \\ Department of Physics and Research Laboratory of Electronics, \\ Massachusetts Institute of Technology, Cambridge, MA 02139, USA
}

\begin{abstract}
The coherent and collective nature of Bose-Einstein condensate can enhance or suppress physical processes. Bosonic stimulation enhances scattering in already occupied states which leads to atom amplification, and the suppression of dissipation leads to superfluidity. In this paper, we review several experiments where suppression and enhancement have been observed and discuss the common roots of and differences between these phenomena.
\end{abstract}

When a gas of bosonic atoms is cooled below the transition temperature of BoseEinstein condensation, it profoundly changes its properties. The appearance of a macroscopically occupied quantum state leads to a variety of new phenomena which set quantum fluids apart from all other substances. Fritz London even called them the fourth state of matter [1].

Many of the key concepts in quantum fluids were derived from studying the weakly interacting Bose gas, for which rigorous theoretical treatments were possible $[2,3]$. In 1995, with the discovery of BEC in a dilute gas of alkali atoms [4-6], it became possible to study such a system experimentally . The theoretical framework connects the observed equilibrium and dynamic properties to the presence of longrange order and low-lying collective excitations [7].

Many special properties of Bose condensates involve the suppression or enhancement of physical processes. Our recent experiments include the suppression and enhancement of elastic collisions of impurity atoms [8], the suppression of dissipation due to superfluidity $[9,10]$, and the suppression [11] and enhancement of light scattering [12]. Bosonically enhanced Rayleigh scattering was used to amplify either atoms [13] or light [14] in a condensate dressed by laser light. We review these experiments and discuss the properties of the Bose condensate which lead to enhancement and suppression. 


\section{SCATTERING OF LIGHT AND MASSIVE PARTICLES}

Before we discuss light scattering and collisions in a BEC, we want to derive some simple general expressions based on Fermi's golden rule which will be useful to see the similarities and differences between the different processes.

When a condensate scatters a photon or material particle, the scattering is described by the Hamiltonian

$$
\mathcal{H}^{\prime}=C \sum_{k, l, m, n} \hat{c}_{l}^{\dagger} \hat{a}_{n}^{\dagger} \hat{c}_{k} \hat{a}_{m} \delta_{l+n-k-m}
$$

Here $\hat{c}_{k}\left(\hat{c}_{k}^{\dagger}\right)$ is the destruction (creation) operator for the scattered particles, and $\hat{a}_{k}\left(\hat{a}_{k}^{\dagger}\right)$ is the destruction (creation) operator for atomic plane waves of wavevector k. The strength of the coupling is parametrized by the coefficient $C$.

We consider the scattering process where a system with $N_{0}$ atoms in the condensate ground state and $N_{q}$ quasi-particles with wavevector $\mathbf{q}$ scatters particles with incident momentum $\mathbf{k}$ into a state with momentum $\mathbf{k}-\mathbf{q}$. The initial and final states are

$$
\begin{aligned}
|i\rangle & =\left|n_{k}, n_{k-q} ; N_{0}, N_{q}\right\rangle \\
|f\rangle & =\left|n_{k}-1, n_{k-q}+1 ; N_{0}-1, N_{q}+1\right\rangle
\end{aligned}
$$

respectively, where $n_{k}$ denotes the population of scattering particles with wavevector $\mathbf{k}^{1}$. It should be emphasized that, due to the interatomic interactions, the quasi-particles with occupation $N_{q}$ are not the plane waves created by the operator $\hat{a}_{q}^{\dagger}$, but the quanta of collective excitations with wavevector $\mathbf{q}$.

The square of the matrix element $M_{1}$ between the initial and final state is

$$
\begin{aligned}
& \left|M_{1}\right|^{2}=\left|\left\langle f\left|\mathcal{H}^{\prime}\right| i\right\rangle\right|^{2} \\
& =|C|^{2}\left|\left\langle N_{0}=N-1, N_{q}=1\left|\hat{\rho}^{\dagger}(\vec{q})\right| N_{0}=N, N_{q}=0\right\rangle\right|^{2}\left(N_{q}+1\right)\left(n_{k-q}+1\right) n_{k}
\end{aligned}
$$

where $\hat{\rho}(\mathbf{q})=\sum_{m} \hat{a}_{m+q}^{\dagger} \hat{a}_{m}$ is the Fourier transform of the atomic density operator at wavevector $\vec{q}$.

The static structure factor of the condensate is $S(q)=\left\langle g\left|\hat{\rho}(\mathbf{q}) \hat{\rho}^{\dagger}(\mathbf{q})\right| g\right\rangle / N$ where $|g\rangle=\left|N_{0}=N, N_{q}=0\right\rangle$ is the BEC ground state. We then obtain for the scattering matrix element $M_{1}$

$$
\left|M_{1}\right|^{2}=|C|^{2} S(q)\left(N_{q}+1\right)\left(n_{k-q}+1\right) N_{0} n_{k}
$$

The scattering rate $W_{1}$ for the process $\left|n_{k}, n_{k-q} ; N_{0}, N_{q}\right\rangle \rightarrow \mid n_{k}-1, n_{k-q}+1 ; N_{0}-$ $\left.1, N_{q}+1\right\rangle$ follows from Fermi's golden rule as

1) This choice of final states implies that we neglect scattering between quasi-particles and consider only processes involving the macroscopically occupied zero-momentum state of the condensate. Formally, we replace the Hamiltonian (Eq. 1) by $C \sum_{k, q}\left(\hat{c}_{k-q}^{\dagger} \hat{a}_{q}^{\dagger} \hat{c}_{k} \hat{a}_{0}+\hat{c}_{k-q}^{\dagger} \hat{a}_{0}^{\dagger} \hat{c}_{k} \hat{a}_{-q}\right)$. 


$$
W_{1}=\frac{2 \pi}{\hbar}\left|M_{1}\right|^{2} \delta\left(E_{k}-E_{k-q}-\hbar \omega_{q}^{B}\right)
$$

where $E_{k}$ is the energy of the incident particle with momentum $\mathbf{k}$, and $\hbar \omega_{q}^{B}$ is the energy of quasi-particles with momentum $\mathbf{q}$ (which we will later obtain from Bogoliubov theory). To obtain the net scattering rate, one has to include the reverse process $\left|n_{k}, n_{k-q} ; N_{0}, N_{q}\right\rangle \rightarrow\left|n_{k}+1, n_{k-q}-1 ; N_{0}+1, N_{q}-1\right\rangle$ by which atoms scatter back into the condensate. The square of the matrix element $M_{2}$ for this process is $|C|^{2} S(q) N_{q} n_{k-q}\left(N_{0}+1\right)\left(n_{k}+1\right)$. The net rate $W_{+}$of scattering atoms from the condensate into the quasi-particle mode $\mathbf{q}$ is the difference of the two partial rates $W_{+}=W_{1}-W_{2}$. Assuming $N_{0} \gg 1$ (i.e. $N_{0}+1 \approx N_{0}$ ), we obtain for the net rate

$$
W_{+}=\frac{2 \pi}{\hbar}|C|^{2} S(q) N_{0}\left[n_{k}\left(N_{q}+n_{k-q}+1\right)-N_{q} n_{k-q}\right] \delta\left(E_{k}-E_{k-q}-\hbar \omega_{q}^{B}\right)
$$

For large $n_{k}$ (e.g. a laser beam illuminating the condensate) the dominant bosonic stimulation term $\left(N_{q}+n_{k-q}+1\right)$ is approximately $(\tilde{N}+1)$ with $\tilde{N}=\max \left(N_{q}, n_{k-q}\right)$. This illustrates that there is no bosonic stimulation of the net rate by the least populated final state. With the dynamic structure factor $S(\mathbf{q}, \omega)=S(q) \delta\left(\omega-\omega_{q}^{B}\right)$ Eq. 6 simplifies to

$$
W_{+} / N_{0}=\frac{2 \pi}{\hbar^{2}}|C|^{2} n_{k} S(\mathbf{q}, \omega)\left(N_{q}+n_{k-q}+1\right)
$$

The rate $W_{+}$in Eq. 6 is the rate for the Stokes process where $E_{k}>E_{k-q}$. Momentum transfer $\mathbf{q}$ to the condensate is also possible as an anti-Stokes process where a quasi-particle with momentum $\mathbf{- q}$ is scattered into the condensate, and the scattered particle gains energy. The net rate $W_{-}$for this process is obtained in an analogous way as

$$
W_{-}=\frac{2 \pi}{\hbar}|C|^{2} S(q) N_{0}\left[n_{k}\left(N_{-q}-n_{k-q}\right)-\left(N_{-q}+1\right) n_{k-q}\right] \delta\left(E_{k}-E_{k-q}+\hbar \omega_{q}^{B}\right) .
$$

The net scattering rates in Eqs. 6 and 8 are the product of three terms.

- The static structure factor $S(q)$ represents the squared matrix element for the condensate to absorb momentum q.

- The delta function denotes the density of final states.

- The bosonic stimulation term represents stimulation by the occupation in the final state either of the scattering particles or the condensate.

The interplay of these three terms is responsible for the suppression and enhancement of physical processes in a condensate. The properties of the condensate as an intriguing many-body system are reflected in the structure factor. In section II, we discuss its measurement through stimulated light scattering. The density of states is responsible for superfluidity because it vanishes for initial velocities of the incident particles which are smaller than the Landau critical velocity (Sections III and IV). Finally, bosonic stimulation by the occupancy $N_{q}$ of final states was responsible for superradiance, matter wave amplification and optical amplification in a condensate (Section V). 


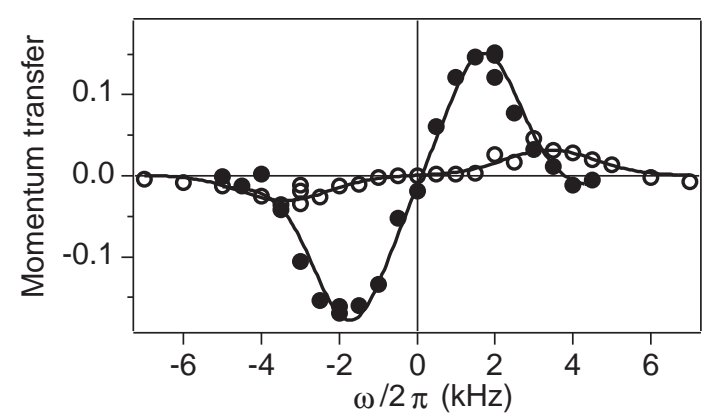

FIGURE 1. Bragg scattering of phonons and of free particles. Momentum transfer per particle, in units of $\hbar q$, is shown vs. the frequency difference $\omega / 2 \pi$ between the two Bragg beams. The two spectra were taken at different atomic densities. The open symbols represent the phonon excitation spectrum for a trapped condensate (at a chemical potential $\mu / h=9.2 \mathrm{kHz}$, much larger than the free recoil shift of $\approx 1.4 \mathrm{kHz}$ ). Closed symbols show the free-particle response of a twenty-three times more dilute (ballistically expanded) cloud. Lines are fits to the difference of two Gaussian line shapes representing excitation in the forward and backward directions. See Refs. [11,16] for more details. Figure is taken from Ref. [11].

\section{DETERMINATION OF THE STATIC STRUCTURE FACTOR BY BRAGG SPECTROSCOPY}

The BEC matrix element or the structure factor in Eqs. 6 and 8 can be directly determined experimentally by stimulated light scattering. The density of states (the $\delta$ function in Eq. 6) does not restrict the scattering since the photon energy is much higher than the quasi-particle energy. As a result, photons can be scattered into the full solid angle and provide the necessary recoil energy of the atom by a small change $\left(\approx 10^{-9}\right)$ in the frequency of the scattered photon.

When the condensate is illuminated by two strong laser beams with wavevectors $\mathbf{k}$ and $\mathbf{k}-\mathbf{q}$ and a difference frequency $\omega$, the rate $W$ of transferring photons from one beam to the other is

$$
W / N_{0}=\left(W_{+}+W_{-}\right) / N_{0}=2 \pi \omega_{R}^{2} S(q)\left(\delta\left(\omega-\omega_{q}^{B}\right)-\delta\left(\omega+\omega_{q}^{B}\right)\right)
$$

where the two-photon Rabi frequency $\omega_{R}$ is given by $\omega_{R}^{2}=|C|^{2} n_{k} n_{k-q} / \hbar^{2}$. When $\omega$ is scanned the "spectrum" of a condensate consists of two peaks at $\pm \omega_{q}^{B}$ (Fig. 1 ). The strength (or integral) of each peak corresponds to $S(q)$. We refer to this method as Bragg spectroscopy since the basic process is Bragg scattering of atoms from an optical standing wave [15].

We observed that the scattering rate was strongly suppressed when the momentum transfer $\hbar \mathbf{q}$ became smaller than the speed of sound $c$ in the condensate, i.e., when the light scattering excited a phonon and not a free particle. These observations are in agreement with the Bogoliubov theory, which obtains the elementary excitations or Bogoliubov quasiparticles as eigenstates of the Hamiltonian (Eq. 1). A quasi-particle with wavevector $q$ is annihilated by the Bogoliubov operator 
$\hat{b}_{q}=u_{q} \hat{a}_{q}^{\dagger}+v_{q} \hat{a}_{-q}$, where $u_{q}=\cosh \phi_{q}, v_{q}=\sinh \phi_{q}$ and $\tanh 2 \phi_{q}=\mu /\left(\hbar \omega_{q}^{0}+\mu\right)$. Its energy is given by

$$
\hbar \omega_{q}^{B}=\sqrt{\hbar \omega_{q}^{0}\left(\hbar \omega_{q}^{0}+2 \mu\right)}
$$

where $\hbar \omega_{q}^{0}=\hbar^{2} q^{2} / 2 m$ and $\mu$ is condensate's chemical potential. Expressing $\hat{\rho}(\mathbf{q})$ by Bogoliubov operators leads to an expression for the static structure factor $S(q)=$ $\left(u_{q}-v_{q}\right)^{2}$. The static structure factor tends to $S(q) \rightarrow \hbar q / 2 m c$ and vanishes in the long wavelength limit, as required of a zero-temperature system with finite compressibility [17].

This reveals a remarkable many-body effect. For free particles, the matrix element for momentum transfer is always 1 , which reflects the fact that the operator $e^{\mathbf{i q} \cdot \mathbf{r}}$ connects an initial state with momentum $\mathbf{p}$ to a state with momentum $\mathbf{p}+\hbar \mathbf{q}$ with unity overlap. For an interacting Bose-Einstein condensate, this overlap vanishes in the long-wavelength limit. As we have discussed in previous publications $[11,16]$, this suppression of momentum transfer is due to an destructive interference between the two pathways for a condensate to absorb momentum $\mathbf{q}$ and create a quasi-particle: one pathway annihilates an admixture with momentum $-\mathbf{q}$, the other creates an admixture at momentum $+\mathbf{q}$.

The leading terms in stimulated light scattering do not depend on temperature. The rates $W_{+}$and $W_{-}$(Eqs. 6 and 8 ) are independent of the thermally excited population of quasiparticles $N_{q}$ and $N_{-q}$ in the limit of large $n_{k}, n_{k-q} \gg N_{ \pm q}$, i.e. when the scattering is induced by a second laser beam. For spontaneous scattering $\left(n_{k-q}=0\right)$, one obtains for the total scattering rate $W$ instead of Eq. 9

$$
W / N_{0}=\frac{2 \pi}{\hbar^{2}}|C|^{2} S(q) n_{k}\left[\left(1+N_{q}\right)\left(\delta\left(\omega-\omega_{q}^{B}\right)+N_{-q} \delta\left(\omega+\omega_{q}^{B}\right)\right)\right] .
$$

Absorption of and bosonic stimulation by thermally excited quasi-particles become important when the temperature is comparable to or larger than the quasi-particle energy $\hbar \omega_{q}^{B}$. Eq. 11 is proportional to the temperature dependent dynamic structure factor $S_{T}(\mathbf{q}, \omega)[18]$.

\section{SUPPRESSION OF IMPURITY COLLISIONS}

The key difference between the scattering of light and massive particles (or impurities) is their energy-momentum dispersion relation. The dispersion relation for impurities is $E_{k}=(\hbar k)^{2} / 2 M$, whereas for light, $E_{k}=\hbar k c_{l}$ with $c_{l}$ denoting the speed of light. This difference is responsible for the complete suppression of impurity scattering at low velocities. For simplicity, we assume equal mass $M$ for the impurity and condensate atoms.

Energy-momentum conservation, the delta function in Eq. 6, requires $E_{k}-E_{k-q}=$ $\hbar \omega_{q}^{B}$. For impurity particles, the l.h.s. is always less than $v \hbar q$, where $v=\hbar k / M$ is the initial velocity of the impurities. Thus, collisions with the condensate are only 


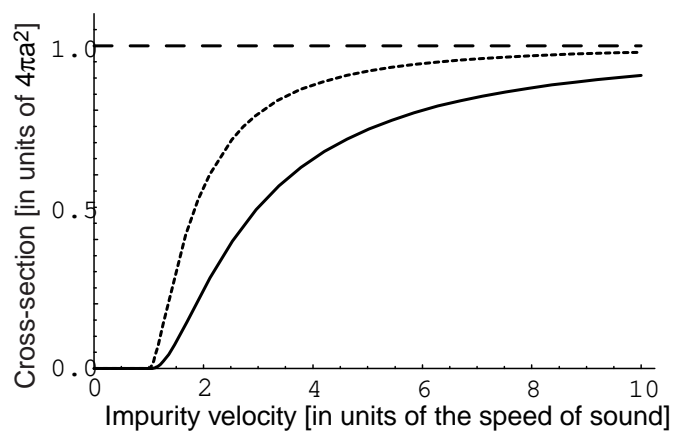

FIGURE 2. Suppression of collisions. Shown is the suppression factor as a function of the impurity velocity (normalized by the speed of sound $c$, solid line). The dotted line represents the suppression due to phase-space restriction alone (i.e., setting the structure factor $S(q)=1$ ).

possible, if this maximum energy transfer is sufficient to excite a quasi-particle, i.e., $v>\min \left(\omega_{q}^{B} / q\right)=v_{L}$, where $v_{L}$ is the Landau critical velocity [19] for superfluidity below which no dissipation occurs because the density of final states vanishes.

For the excitation spectrum of the condensate (Eq. 10), the Landau velocity is the Bogoliubov speed of sound $v_{L}=c=\sqrt{\mu / M}$. Impurity particles moving below this speed cannot dissipate energy in collisions. In contrast, for photons, $\mathrm{d} E_{k} / \mathrm{d}(\hbar k)=c_{l} \gg c$, and scattering is always possible.

In the perturbative limit (no stimulation by the final occupation), the total rate of scattering $\Gamma$ is given by integrating Eq. 6 over all possible momentum transfers.

$$
\begin{aligned}
\Gamma & =\frac{2 \pi|C|^{2}}{\hbar^{2}} N_{0} \sum_{\mathbf{q}} S(q) \delta\left(\frac{\hbar \mathbf{k} \cdot \mathbf{q}}{M}-\frac{\hbar q^{2}}{2 M}-\omega_{q}^{B}\right) \\
& =\left(N_{0} / V\right)\left(\frac{2 \hbar a}{M}\right)^{2} \int d q d \Omega q^{2} S(q) \delta\left(\frac{\hbar k q \cos \theta}{M}-\frac{\hbar q^{2}}{2 M}-\omega_{q}^{B}\right) \\
& =2 \pi\left(N_{0} / V\right)\left(\frac{2 \hbar a}{M}\right)^{2} \frac{1}{v} \int_{0}^{Q} d q q S(q) \\
& =\left(N_{0} / V\right) \sigma(\eta) v,
\end{aligned}
$$

where $\hbar Q=M v\left(1-1 / \eta^{2}\right)$ is the maximum possible momentum transfer, and $\eta=v / c$ must be larger than 1 . We have used $C=4 \pi \hbar^{2} a / M V$ for $s$-wave scattering between condensate and the impurity particles where $a$ denotes the scattering length and $V$ the condensate volume. The collision cross section is $\sigma(\eta)=\sigma_{0} F(\eta)$ where $\sigma_{0}=4 \pi a^{2}$. For $\eta<1, F(\eta)=0$ and for $\eta>1, F(\eta)=1-1 / \eta^{4}-\log \left(\eta^{4}\right) / \eta^{2}$.

The suppression factor $F(\eta)$ is determined by two factors: the phase space restriction due to the delta function in Eq. 12, and additional suppression at low momentum transfers by the structure factor of the condensate. For decreasing velocity, the possible scattering angles become restricted to a forward scattering cone, which shrinks to zero solid angle at the Landau critical velocity. A graph of the suppression factor as function of impurity velocity is shown in Fig. 2. 


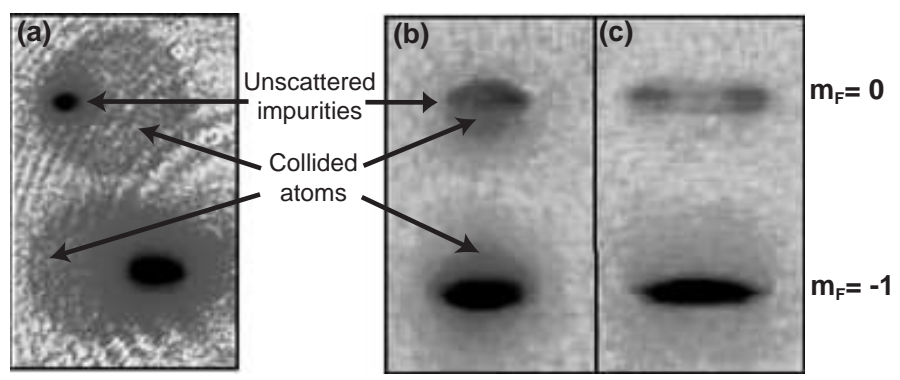

FIGURE 3. Observation of elastic collisions between the condensate and impurity atoms. (a) Impurities traveling at $6 \mathrm{~cm} / \mathrm{s}$ along the radial axis (to the left in images) were scattered into a $s$-wave halo. Absorption image after $50 \mathrm{~ms}$ of time-of-flight shows the velocity distribution after collisions between the condensate (bottom) and the outcoupled $m_{F}=0$ atoms (top), spatially separated by a Stern-Gerlach type magnetic field gradient. The collisional products are distributed over a sphere in momentum space. The image is $4.5 \times 7.2 \mathrm{~mm}$. (b) Similar image as (a) shows the collisional products (arrow) for impurity atoms (top) traveling at $7 \mathrm{~mm} / \mathrm{s}$ along the condensate axis (upward in image). For this image, $v_{g} / c=2.7$ (see text). Collisions are visible below the unscattered impurities. (c) Similar image as (b) with $v_{g} / c=1.6$. Collisions are suppressed. The outcoupled atoms were distorted by mean-field repulsion. The images are $2.0 \times 4.0 \mathrm{~mm}$.

Experimentally, the Landau critical velocity can usually only be observed by moving microscopic particles through the superfluid which do not create a macroscopic flow pattern. Studies of superfluidity with microscopic objects were pursued in liquid ${ }^{4} \mathrm{He}$ by dragging negative ions through pressurized ${ }^{4} \mathrm{He}[20,21]$, and by scattering ${ }^{3} \mathrm{He}$ atoms off superfluid ${ }^{4} \mathrm{He}$ droplets [22].

To study the effects of impurities interacting with the condensate, we created microscopic impurity atoms using a stimulated Raman process which transferred a small fraction of the condensate atoms in the $\left|F=1, m_{F}=-1\right\rangle$ hyperfine state into an untrapped hyperfine state $\left|F=1, m_{F}=0\right\rangle$ with a well-defined initial velocity [23]. The initial velocity could be adjusted between zero and two recoil velocities by varying the angle between the two Raman beams. As these impurities traversed the condensate, they collided with the stationary condensate, resulting in a redistribution of the impurity momenta which was detected by a time-of-flight analysis [8] (see Fig. 3).

To probe for the suppression of collisions, one has to vary the impurity velocity around the speed of sound. For that, we produced impurity atoms at low velocities $(7 \mathrm{~mm} / \mathrm{s})$ and varied the speed of sound by changing the condensate density. The small axial velocity imparted by Raman scattering allowed us to identify products of elastic collisions in time-of-flight images (Fig. 3b, c) since collisions with the stationary condensate redistributed the impurity atoms toward lower axial velocities. However, the impurity velocity was predominantly determined by the gravitational acceleration $g$, which imparted an average velocity of $v_{g}=\sqrt{2 g z_{c}}$ where $z_{c}$ is the Thomas-Fermi radius of the condensate in the $z$-direction. Thus, the effect of superfluidity on impurity scattering depends primarily on the parameter $\bar{\eta}=v_{g} / c$ which is the ratio of the typical impurity velocity $v_{g}$ to the speed of sound $c$ at the 


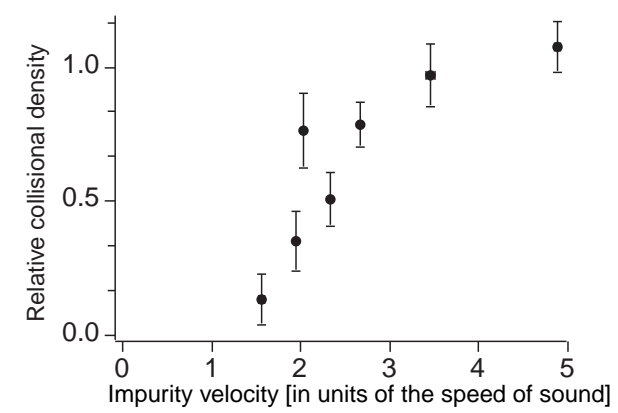

FIGURE 4. Onset of superfluid suppression of collisions. Shown is the observed collisional density normalized to the predicted one in the limit of high velocities as a function of $\bar{\eta}=v_{g} / c$, which is a measure of the impurity velocity in units of the condensate's speed of sound. The error bars represent the statistical uncertainty. Data is taken from Ref. [8]

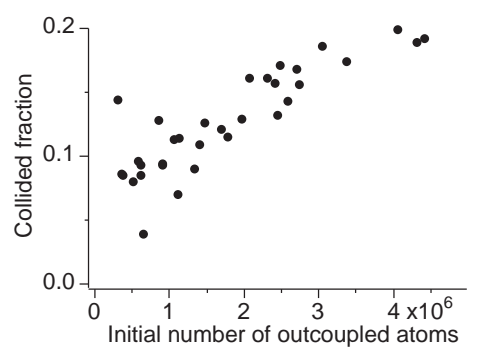

FIGURE 5. Collective amplified elastic scattering in a Bose-Einstein condensate. Shown is the fraction of collided atoms vs. the number of outcoupled atoms. For this data, $v_{g} / c=4.9$ and the chemical potential was $1.8 \mathrm{kHz}$. Figure is taken from Ref. [8]

center of the condensate.

A time-of-flight analysis of impurity scattering for the case of a low-density condensate (small $c$ ) and large condensate radius (large $v_{g}$ ) is shown in Fig. $3 \mathrm{~b}$. The effect of collisions is clearly visible with about $20 \%$ of the atoms scattered to lower axial velocities (below the unscattered impurities in the image). In contrast, in the case of tight confinement, the condensate density is higher (larger $c$ ) and its radius is smaller (smaller $v_{g}$ ), and the collision probability is greatly suppressed due to superfluidity (Figs. 3c and 4).

The bosonic stimulation factor in Eq. 6 is relevant if the final states are populated, either by scattering or thermally. We observed that the fraction of collided atoms increased with the number of outcoupled impurities (Fig. 5). For a large outcoupled fraction, population $n_{k-q}$ and $N_{q}$ is built up in the final states and stimulates further scattering. This collisional amplification is not directional, and is similar to the recently observed optical omnidirectional superfluoresence [24].

Gain of momentum and thus transfer of energy from the condensate to the impurity atoms is impossible at zero temperature, but may happen at finite temperature due to the presence of thermal excitations (the $N_{-q}$ term in Eq. 8) ${ }^{2}$. Thus finite

2) We are grateful to S. Stringari for pointing out the importance of finite-temperature effects. 


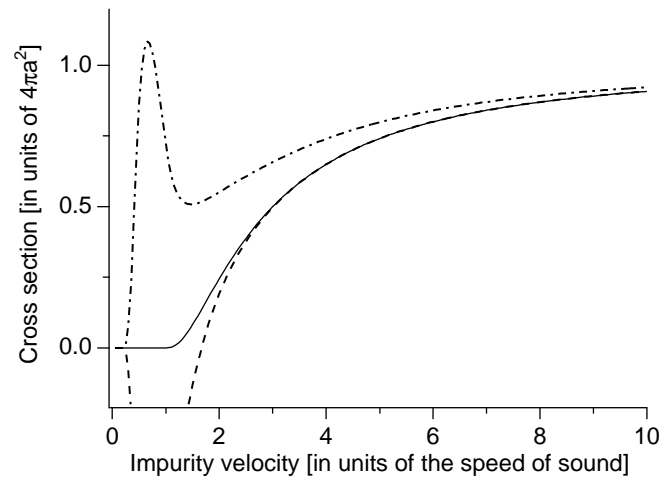

FIGURE 6. Temperature dependent cross-section vs. impurity velocity. Shown is the cross-section at zero temperature (solid line) and at a finite temperature $k T=\mu$ which is typical for our experimental conditions (dash-dotted line). The finite temperature cross-section includes collisions involving thermally occupied quasi-particles where the impurities lose or gain energy. In the experiment, we measured the number of impurities which lost its energy minus the number which gained energy. Thus, the experimental measured cross-sections (Fig. 4) should be compared to $\sigma_{\text {coll,loss }}-\sigma_{\text {coll,gain }}$ (dashed line).

temperature enhances the elastic cross section by two effects: Absorption of quasiparticles (anti-Stokes process) and stimulation of momentum transfer by the final state population (Stokes process).

Fig. 6 shows the dramatic variation of the elastic scattering cross section with temperature. However, the finite temperature did not affect our data in a major way: Due to gravitational acceleration we couldn't probe the velocity regime well below the Landau critical velocity where only thermally assisted collisions are possible. Furthermore, when we counted the number of collided atoms we had to use a background subtraction method where we subtracted the small signal of the energy gain collisions from the energy loss collisions (see Ref. [8] for details), thus cancelling most of the finite-temperature effects.

\section{SUPPRESSION OF DISSIPATION FOR A MOVING MACROSCOPIC OBJECT}

So far, we have discussed the suppression and enhancement of microscopic processes (light scattering and impurity collisions). The suppression of dissipation is even more dramatic on the macroscopic scale. The flow of liquid ${ }^{4} \mathrm{He}$ and the motion of macroscopic objects through it are frictionless below a critical velocity [25]. Recently, we have explored such frictionless flow in a gaseous BEC $[9,10]$.

The microscopic and macroscopic cases bear many parallels. The onset of scattering or dissipation has two requirements: one needs final states which conserve energy and momentum, and an overlap matrix element which populates these states. In the case of macroscopic flow, the first requirement leads to a critical velocity 
for vortex creation and the second requirement addresses the nucleation process of vortices.

The Landau criterion for superfluidity shows that excitations with momentum $p$ and energy $E(p)$ are only possible when the relative velocity between the fluid and the walls or a macroscopic object exceeds the Landau critical velocity $v_{L}$ which is given by $v_{L}=\min (E(p) / p$ ) (see e.g. $[2,25]$. A similar criterion applied to vortex formation yields

$$
v_{c}=\frac{E_{\mathrm{vortex}}}{I_{\mathrm{vortex}}} \sim \frac{\hbar}{M d} \ln \left(\frac{d}{\xi}\right)
$$

where $I=\int \mathrm{Pd}^{3} r$ is the integrated momentum of the vortex ring or line pair, $E$ is its total energy, $d$ is the dimension of the container, and $\xi$ the core radius of a vortex which in the case of dilute gases is the healing length $\xi=1 / \sqrt{8 \pi n a}$. Ref. [25] derived Eq. 13 for vortex rings with a maximum radius d, Ref. [26] looked at pairs of line vortices at distance $d$. Feynman [27] found a similar result for superflow through a channel of diameter $d$.

A similar result is obtained for a Bose condensed system placed under uniform rotation with angular velocity $\Omega$. A vortex becomes energetically allowed when its energy $E^{\prime}$ in the rotating frame drops to zero,

$$
E^{\prime}=E-\Omega L=0
$$

where $E$ and $L$ are the energy and angular momentum in the laboratory frame. This defines a critical angular velocity below which a vortex cannot be sustained due to conservation of angular momentum and energy [28]:

$$
\Omega_{c}=\frac{E_{\mathrm{vortex}}}{L_{\mathrm{vortex}}} \sim \frac{\hbar}{M d^{2}} \ln \left(\frac{d}{\xi}\right) .
$$

The critical velocity at the wall of the rotating container, $v_{c}=d \Omega_{c}$, agrees with Eq. 13.

However, Eqs. 13 and 15 only reflect the energy and momentum required to generate vortices, and do not take into account the nucleation process. If the scattering particle is macroscopic in size, the coupling is between the ground state and a state containing a vortex. Populating such a state requires nucleation of the vortex by the perturbing potential, which usually does not occur until higher velocities are reached than those predicted by Eqs. 13 and 15. The other option, the formation of the vortex by macroscopic quantum tunneling between the two states is an extremely slow process. In recent experiments in which a Bose condensate was placed in a rotating potential, the critical angular velocity for the formation of a single vortex was observed to be 1.6 times higher than the value given by Eq. 15 [29]. This discrepancy may be due to a nucleation barrier associated with the excitation of surface modes, as some authors have recently suggested [30,31]. 
To study frictionless flow in a Bose-condensate, we focused a blue-detuned 514 $\mathrm{nm}$ Argon laser beam onto the sodium condensate, which repelled atoms from the focus. The laser beam was scanned back and forth along the axial direction of the condensate, creating a moving "hole" that simulated a macroscopic object. Rapid sequence phase-contrast imaging allowed us to directly measure the flow pattern of the superfluid around the moving laser beam.

For a weakly interacting Bose-condensed gas at density $n(\mathbf{r})$ and chemical potential $\mu$, pressure is identical to the mean-field energy density $P=\mu(\mathbf{r}) n(\mathbf{r}) / 2[7]$. A drag force arises due to the pressure difference across the moving object. The chemical potential is given by $\mu(\mathbf{r}, t)=g n(\mathbf{r}, t)$, where $g=4 \pi \hbar^{2} a / M$ is the strength of two-body interactions. The drag force $F$ is given by

$$
F \simeq g S n \Delta n=S \mu \Delta \mu / g
$$

where $\Delta n$ and $\Delta \mu$ are the differences in density and chemical potential across the stirring object, and $S$ the surface area the macroscopic object presents to the condensate.
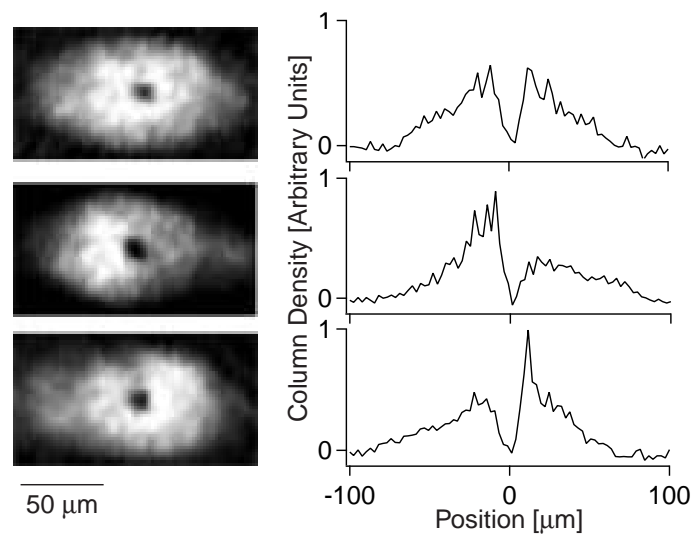

FIGURE 7. Pressure difference across a laser beam moving through a condensate. On the left side in situ phase contrast images of the condensate are shown, strobed at each stirring half period: beam at rest (top); beam moving to the left (middle) and to the right (bottom). The profiles on the right are horizontal cuts through the center of the images. The stirring velocity and the maximum sound velocity were $3.0 \mathrm{~mm} / \mathrm{s}$ and $6.5 \mathrm{~mm} / \mathrm{s}$ respectively. Figure is taken from Ref. [10].

If the laser beam is stationary, or moves slowly enough to preserve the superfluid state of the condensate, there will be no gradient in the chemical potential across the laser focus, and therefore zero force according to Eq. 16. A drag force between the moving beam and the condensate is indicated by an instantaneous density distribution $n(\mathbf{r}, \mathbf{t})$ that is distorted asymmetrically with respect to the laser beam. Fig. 7 shows phase contrast images strobed at half the stirring period (where the laser beam is in the center of the condensate). A bow wave and stern wave form in front of and behind the moving laser beam, respectively. We define the asymmetry $A$ as the relative difference between the peak column densities in front $\left(\tilde{n}^{f}\right)$ 
and behind $\left(\tilde{n}^{b}\right)$ the laser beam $A=2\left(\tilde{n}^{f}-\tilde{n}^{b}\right) /\left(\tilde{n}^{f}+\tilde{n}^{b}\right)$. The asymmetry $A$ is proportional to the drag force $F$.

In Fig. 8 we show measurements of the asymmetry for two maximum densities $n_{0}$ of $9 \times 10^{13}$ and $1.9 \times 10^{14} \mathrm{~cm}^{-3}$. In each data set there is a threshold velocity $v_{c}$ below which the drag force is negligible, and this threshold increases at higher density. Its value is close to $0.1 c$ for both data sets, where $c$ is the sound velocity. Above this critical velocity, the drag force increases monotonically, with a larger slope at low density. In addition, the heating rate due to friction against the laser beam was directly measured through time of flight absorption imaging $[9,10,32]$ and found to be in good agreement with the value $\mathbf{F} \cdot \mathbf{v}$, with $\mathbf{F}$ estimated from Eq. 16.

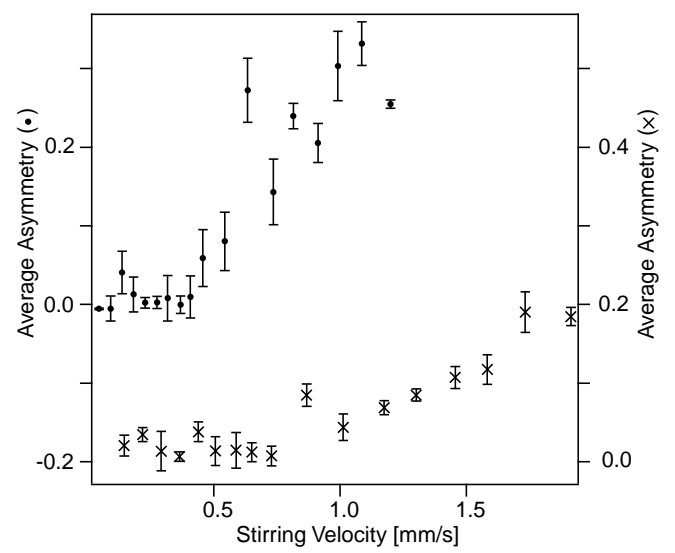

FIGURE 8. Density dependence of the critical velocity. The onset of the drag force is shown for two different condensate densities, corresponding to maximum sound velocities of $4.8 \mathrm{~mm} / \mathrm{s}$ $(\bullet$, left axis) and $7.0 \mathrm{~mm} / \mathrm{s}(\times$, right axis). The stirring amplitudes are $29 \mu \mathrm{m}$ and $58 \mu \mathrm{m}$, respectively. The two vertical axes are offset for clarity. The bars represent statistical errors. Figure is taken from Ref. [10].

The observed critical velocity may be related to the formation of vortices. An estimate based on Eq. 13 for typical experimental parameters in sodium $(d=10 \mu \mathrm{m}$, peak density $\left.n_{0}=1.5 \times 10^{14} \mathrm{~cm}^{-3}, a=2.75 \mathrm{~nm}\right)$ yields $v_{c} \simeq 1.0 \mathrm{~mm} / \mathrm{s}$, close to the experimental observations. However, Eq. 13 depends only weakly on the speed of sound, through the logarithmic dependence on the healing length $\xi$. In contrast, our measurements show an approximate proportionality to the sound velocity [10], suggesting that vortex nucleation, determines the onset of dissipation.

Time-dependent simulations of the Gross-Pitaevskii equation show the formation of vortex line pairs, above a critical velocity which is close to the observed value [33]. Several authors have emphasized the role of locally supersonic flow around the laser beam in the nucleation of vortices. [33-35]. In one theoretical model [34], the vortices are emitted periodically at a rate that increases with velocity, and reduce the pressure gradient across the object. The predicted heating rate $[34,36]$ is in rough agreement with the data. Moreover, this model also predicts that the slope 
of the asymmetry should increase at lower density, in accord with our observations.

\section{AMPLIFICATION OF LIGHT AND ATOMS}

Spontaneous light scattering can be stimulated when the atomic recoil state is already populated (the $N_{q}$ term in Eq. 6). We have explored this process in our studies of superradiance [12], phase-coherent atom amplification [13] and optical amplification [14].

In all these experiments, the condensate was illuminated with a laser beam (mode $k$, also called "dressing beam"). A condensate atom scatters a photon from the laser beam into another mode and receives the corresponding recoil momentum and energy. Injection of atoms or light turns this spontaneous process into a stimulated process and realizes an amplifier for either atoms or light. If atoms are injected, they form a matter wave grating (an interference pattern with the condensate at rest) and this grating diffracts light. The diffraction transfers recoil momentum and energy to the atoms, which results in a growth of the grating and therefore the number of atoms in the recoil mode - this is the intuitive picture for atom gain.

Eq. 6 describes gain for atoms. In the limit of an empty mode for the scattered light $\left(n_{k-q}=0\right)$, one obtains

$$
W_{+}=\frac{2 \pi}{\hbar}|C|^{2} N_{0} n_{k}\left(N_{q}+1\right) \delta\left(E_{k}-E_{k-q}-\hbar \omega_{q}^{B}\right)
$$

For the high momentum transfers considered here (on the order of the photon recoil momentum), $S(q)=1$. Each scattering event which transfers momentum q to the condensate, generates a recoiling atom - therefore the scattering rate $W_{+}$ integrated over all final states for the scattered photon gives the growth rate $\dot{N}_{q}$ for the recoiling atoms:

$$
\dot{N}_{q}=\left(G_{q}-\Gamma_{2, q}\right) N_{q}
$$

with the gain coefficient

$$
G_{q}=R N_{0} \frac{\sin ^{2} \theta_{q}}{8 \pi / 3} \Omega_{q} .
$$

Here $R$ is the rate for single-atom Rayleigh scattering which is proportional to the pump light intensity, $N_{0}$ the number of atoms in the condensate at rest, $\theta_{q}$ the angle between the polarization of the incident light and the direction of the scattered light, and $\Omega_{q}$ the phase-matching solid angle for scattering into mode $q$. In addition, a loss term $\Gamma_{2, q}$ was included which describes the decoherence rate of the matter-wave grating and determines the threshold for exponential growth. It represents the linewidth of the two-photon process generating recoil atoms in mode $q$. 


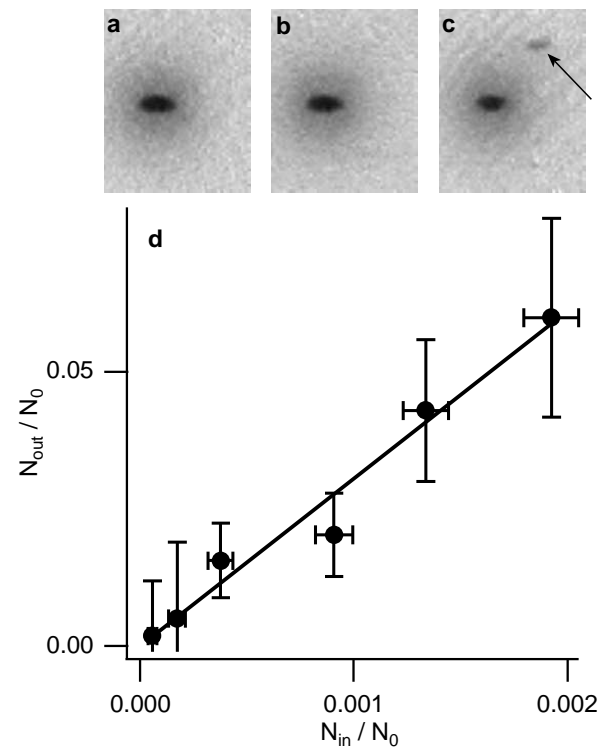

FIGURE 9. Input-output characteristic of the matter-wave amplifier. (a-c) Typical time-of-flight absorption images demonstrating matter wave amplification. The output of the seeded amplifier (c) is clearly visible, whereas no recoiling atoms are discernible in the case without amplification (a) or amplification without the input (b). The size of the images is $2.8 \mathrm{~mm} \times$ $2.3 \mathrm{~mm}$. (d) Output of the amplifier as a function of the number of atoms at the input. A straight line fit shows a number gain of 30. Reprinted by permission from Nature, Ref. [13], copyright 1999 Macmillan Magazines Ltd.

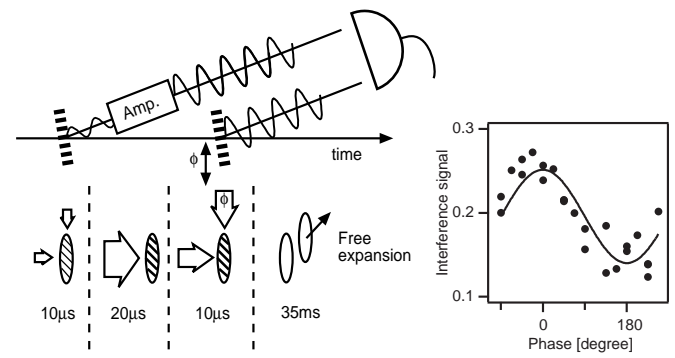

FIGURE 10. Experimental scheme for observing phase coherent matter wave amplification. A small-amplitude matter wave was split off the condensate by applying a pulse of two off-resonant laser beams (Bragg pulse). This input matter wave was amplified by passing it through the condensate pumped by a laser beam. The coherence of the amplified wave was verified by observing its interference with a reference matter wave, which was produced by applying a second (reference) Bragg pulse to the condensate. The interference signal was observed after $35 \mathrm{~ms}$ of ballistic expansion. The fringes on the right side show the interference between the amplified input and the reference matter wave. Reprinted by permission from Nature, Ref. [13], copyright 1999 Macmillan Magazines Ltd. 
Fig. 9 shows the input-output characteristics of the amplifier. The number of input atoms was below the detection limit of our absorption imaging (Fig. 9a). The amplification pulse alone, although above the threshold for superradiance [12], did not generate a discernible signal of atoms in the recoil mode (Fig. 9b). When the weak input matter wave was added, the amplified signal was clearly visible (Fig. 9c). The gain was controlled by the intensity of the pump pulse (see Eq. 19) and typically varied between 10 and 100. Fig. 9d shows the observed linear relationship between the atom numbers in the input and the amplified output with a number gain of 30 .

The phase of the amplified matter wave was determined with an interferometric technique. For this, a reference matter wave was split off the condensate in the same way as the first (input) wave (see Fig. 10). The phase of the reference matter wave was scanned by shifting the phase of the radio-frequency signal that drove the acousto-optic modulator generating the axial Bragg beam. We then observed the interference between the reference and the amplified matter waves by measuring the number of atoms in the recoil mode.

The atom amplification is described by the Hamiltonian (Eq. 1) as a four-wave mixing process between two electromagnetic fields and two Schrödinger fields. The symmetry between light and atoms indicates that a dressed condensate should not only amplify injected atoms, but also injected light. In Ref. [16] we have discussed that matter wave gain and optical gain emerge as two limiting cases, depending on whether the atomic population $N_{q}$ or occupation of the optical mode $n_{k-q}$ dominates the bosonic stimulation term in Eq. 7 . In our experiments, the recoiling atoms move ten orders of magnitude more slowly than the scattered light. Therefore, we always have $N_{q} \gg n_{k-q}$ and $n_{k-q}<1$ and optical stimulation should play no role. Still, as we want to discuss now, the dressed condensate can act as an amplifier for light.

The physical picture behind the optical gain is as follows: If a very weak probe beam is injected into the dressed condensate, it acts together with the dressing beam as a pair of Bragg beams and creates recoiling atoms. Those recoiling atoms move out of the condensate (or decohere) on a time scale $\Gamma_{2}^{-1}$ which is the inverse of the linewidth of the Bragg transition. In steady state, the number of recoiling atoms $N_{q}$ in the volume of the condensate is proportional to the intensity of the probe light. Those recoiling atoms interfere with the condensate at rest and form a diffraction grating which diffracts the dressing beam into the path of the probe light resulting in amplification of the probe light (Fig. 11).

An expression of the gain can be derived in analogy to a fully inverted twolevel system with dipole coupling which would have a gain cross section of $6 \pi \lambda^{2}$ for radiation with wavelength $\lambda(=2 \pi \lambda)$. For the Raman-type system in Fig. 11b, the gain is reduced by the excited state fraction, $R / \Gamma_{1}$ (where $R$ is the Rayleigh scattering rate for the dressing beam and $\Gamma_{1}$ is the linewidth of the single-photon atomic resonance) and increased by $\Gamma_{1} / \Gamma_{2}$, the ratio of the linewidths of the singlephoton and two-photon Bragg resonances. Thus the expected cross-section for gain is 

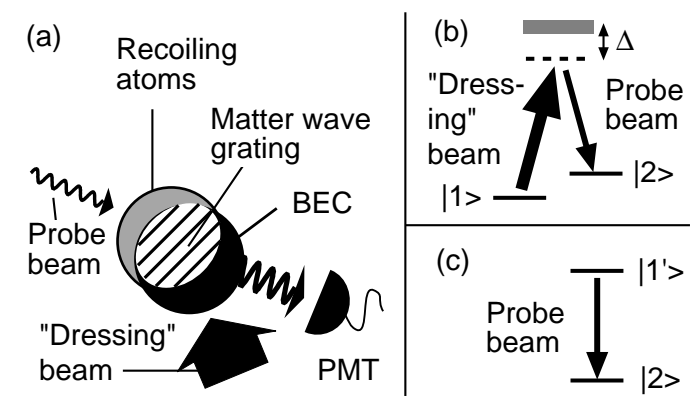

FIGURE 11. Amplification of light and atoms by off-resonant light scattering. (a) The fundamental process is the absorption of a photon from the "dressing" beam by an atom in the condensate (state $|1\rangle$ ), which is transferred to a recoil state (state $|2\rangle$ ) by emitting a photon into the probe field. The intensity in the probe light field was monitored by a photomultiplier. (b) The two-photon Raman-type transition between two motional states $(|1\rangle,|2\rangle)$ gives rise to a narrow resonance. (c) The dressed condensate is the upper state $\left(\left|1^{\prime}\right\rangle\right)$ of a two-level system, and decays to the lower state (recoil state of atoms, $|2\rangle$ ) by emitting a photon. Figure is taken from Ref. [14]

$$
\sigma_{\text {gain }}=6 \pi \lambda^{2} \frac{R}{\Gamma_{2}}
$$

We observed quasi-steady state gain by a factor of up to three. This optical gain has a narrow bandwidth due to the long coherence time of a condensate. The gain represents the imaginary part of the complex index of refraction. A sharp peak in the gain implies a steep dispersive shape for the real part of the index of refraction $n(\omega)$. This resulted in an extremely slow group velocity for the amplified light. Fig. 12 shows that light pulses were delayed by about $20 \mu$ s across the $20 \mu \mathrm{m}$ wide condensate corresponding to a group velocity of $1 \mathrm{~m} / \mathrm{s}$. This is one order of magnitude slower than any value reported previously (see Ref. [37] and references therein).

The (amplitude) gain $g$ for the probe light is related to the matter wave gain $G$

$$
g=1+\frac{n_{0} \sigma_{\text {gain }} l}{2}=1+\frac{G}{\Gamma_{2}}
$$

where $n_{0}$ is the condensate density and $l$ its length. However, when the gain $G$ is above the threshold for superradiance, $G>\Gamma_{2}$ (Eq. 18) the optical gain should diverge: a single recoiling atom created by the probe light and dressing light is exponentially amplified and creates a huge matter wave grating which will diffract the dressing light into the probe light path, thus amplifying the probe light by a large factor. In order to describe this non-linear feedback, one has to use coupled equations for optical gain and atom gain [14]. As a result, one has to replace the loss rate $\Gamma_{2}$ in Eq. 21 by the dynamic rate $\Gamma_{2}-G$ (see Eq. 18)

$$
g=1+\frac{G}{\Gamma_{2}-G}=1+\frac{n_{0} \sigma_{\text {gain }} l}{2} \frac{\Gamma_{2}}{\Gamma_{2}-G}
$$


which agrees with Eq. (21) in the low-intensity limit. By raising the gain over the threshold we could map out the transition from single-atom gain to collective gain [14]. The expansion $g=1+\left(G / \Gamma_{2}\right)+\left(G / \Gamma_{2}\right)^{2}+\ldots$ shows the transition from (linear) single-atom gain to (non-linear) collective gain and illustrates that the dressed condensate is a clean model system for discussing linear and non-linear behavior, optical and atom-optical properties and their interplay.
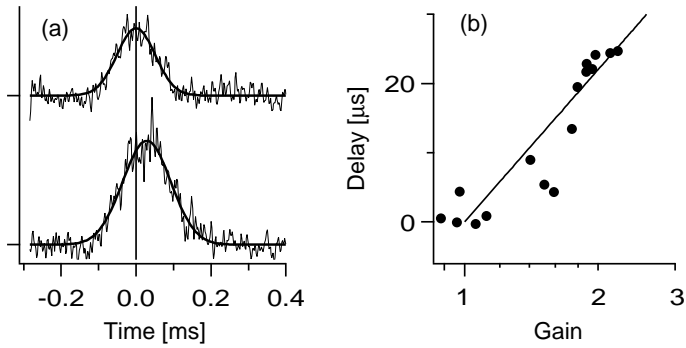

FIGURE 12. Pulse delay due to light amplification. (a) About $20 \mu$ s delay was observed when a Gaussian pulse of about $140 \mu \mathrm{s}$ width and $0.11 \mathrm{~mW} / \mathrm{cm}^{2}$ peak intensity was sent through the dressed condensate (bottom trace). The top trace is a reference taken without the dressed condensate. Solid curves are Gaussian fits to guide the eyes. (b) The observed delay was proportional to the logarithm of the observed gain. Figure is taken from Ref. [14]

\section{DISCUSSION}

This paper has summarized our recent experiments on Bose-Einstein condensation with the unifying theme of suppression and enhancement. Suppression of scattering or dissipation can arise for two different reasons. The phonon and vortex nature of the collective excitations together with energy and momentum conservation allow dissipation only above a critical velocity. In addition, one has to consider the dynamics of the excitation process. For microscopic particles, this is reflected in the matrix element $S(q)$ which characterizes how easily can the condensate absorb momentum. For macroscopic motion, it is reflected in a critical velocity for vortex nucleation. Scattering processes are also enhanced by the population in the final states (bosonic stimulation). Optical stimulation was used in Bragg scattering, and matter wave stimulation led to superradiance and matter wave amplification.

In closing, let us note that there are some subtleties which go beyond the simple picture using rate equations and occupation numbers. A condensate in its ground state is in a coherent superposition state of the zero-momentum state with correlated pairs with momenta $\pm \mathbf{q}$ (the quantum depletion). The population in the quantum depletion can cause bosonic stimulation of spontaneous emission [38]. However, for a scattering situation, there are two bosonically enhanced pathways which destructively interfere (causing $S(q)<1$, Sect. II). Therefore, the concept of bosonic stimulation can be applied to the quantum depletion, but with caution [38]. 
Finally, the matter wave amplification described in Sect. V can be regarded as the self-amplification of a density modulation (caused by matter wave interference between the condensate and the amplified recoiling atoms). A similar amplification can happen for a density modulation in a fermionic gas [39], but, the coherence time of such a density modulation is generally much shorter than in a condensate. This example shows that a more general description of stimulation and amplification has to address the symmetry and coherence of the prepared state. Bosonic quantumdegeneracy is sufficient, but not necessary for stimulated scattering [39].

We are grateful to Axel Görlitz for helpful discussions. This work was supported by NSF, ONR, ARO, NASA, and the David and Lucile Packard Foundation.

\section{REFERENCES}

1. F. London, Superfluids, Vol.II (Dover, New York, 1964).

2. K. Huang, Statistical Mechanics (Wiley, New York, 1987).

3. N. N. Bogoliubov, J. Phys. (USSR) 11, 23 (1947).

4. M. H. Anderson, J. R. Ensher, M. R. Matthews, C. E. Wieman, and E. A. Cornell, Science 269, 198 (1995).

5. K. B. Davis, M.-O. Mewes, M. A. Joffe, M. R. Andrews, and W. Ketterle, Phys. Rev. Lett. 74, 5202 (1995).

6. C. C. Bradley, C. A. Sackett, J. J. Tollet, and R. G. Hulet, Phys. Rev. Lett. 75, 1687 (1995).

7. F. Dalfovo, S. Giorgini, L. P. Pitaevskii, and S. Stringari, Rev. Mod. Phys. 71, 463 (1999).

8. A. P. Chikkatur, A. Görlitz, D. M. Stamper-Kurn, S. Inouye, S. Gupta, and W. Ketterle, Phys. Rev. Lett. 85, 483 (2000).

9. C. Raman, M. Köhl, R. Onofrio, D. S. Durfee, C. E. Kuklewicz, Z. Hadzibabic, and W. Ketterle, Phys. Rev. Lett. 83, 2502 (1999).

10. R. Onofrio, C. Raman, J. M. Vogels, J. Abo-Shaeer, A. P. Chikkatur, and W. Ketterle, Phys. Rev. Lett. 85, 2228 (2000).

11. D. M. Stamper-Kurn, A. P. Chikkatur, A. Görlitz, S. Inouye, S. Gupta, D. E. Pritchard, and W. Ketterle, Phys. Rev. Lett. 83, 2876 (1999).

12. S. Inouye, A. P. Chikkatur, D. M. Stamper-Kurn, J. Stenger, D. E. Pritchard, and W. Ketterle, Science 285, 571 (1999).

13. S. Inouye, T. Pfau, S. Gupta, A. P. Chikkatur, A. Görlitz, D. E. Pritchard, and W. Ketterle, Nature 402, 641 (1999).

14. S. Inouye, R.F.Löw, S. Gupta, T. Pfau, A. Görlitz, T. L. Gustavson, D. E. Pritchard, and W. Ketterle, cond-mat/0006455; Phys. Rev. Lett., in print.

15. J. Stenger, S. Inouye, A. P. Chikkatur, D. M. Stamper-Kurn, D. E. Pritchard, and W. Ketterle, Phys. Rev. Lett. 82, 4569 (1999).

16. D. Stamper-Kurn and W. Ketterle, proceedings of the Les Houches Summer School on Bose-Einstein Condensation in 1999, in print; cond-mat/0005001.

17. P. J. Price, Phys. Rev. 94, 257 (1954). 
18. A. Griffin, Excitations in a Bose-condensed liquid (Cambridge University Press, Cambridge, 1993).

19. L. D. Landau, J. Phys. (USSR) 5, 71 (1941).

20. L. Meyer and F. Reif, Phys. Rev. 123, 727 (1961).

21. D. R. Allum, P. V. E. McClintock, A. Phillips, and R. M. Bowley, Phil. Trans. R. Soc. A 284, 179 (1977).

22. J. Harms and J. P. Toennies, Phys. Rev. Lett. 83, 344 (1999).

23. E. W. Hagley, L. Deng, M. Kozuma, J. Wen, K. Helmerson, S. L. Rolston, and W. D. Phillips, Science 283, 1706 (1999).

24. A. I. Lvovsky and S. R. Hartmann, Phys. Rev. Lett. 82, 4420 (1999).

25. P. Nozières and D. Pines, The Theory of Quantum Liquids (Addison-Wesley, Redwood City, CA, 1990).

26. M. Crescimanno, C. G. Koay, R. Peterson, and R. Walsworth, preprint condmat/0001163; Phys. Rev. A, in print.

27. R. P. Feynman, in Progress in Low Temperature Physics, edited by C. Gorter (NorthHolland, Amsterdam, 1955), Vol. 1, p. 17.

28. E. Lundh, C. J. Pethick, and H. Smith, Phys. Rev. A 55, 2126 (1997).

29. K. W. Madison, F. Chevy, W. Wohlleben, and J. Dalibard, Phys. Rev. Lett. 84, 806 (2000).

30. D. L. Feder, C. W. Clark, and B. I. Schneider, Phys. Rev. A 61, 011601(R) (1999).

31. T. Isoshima and K. Machida, Phys. Rev. A 60, 3313 (1999).

32. C. Raman, R. Onofrio, J. M. Vogels, J. R. Abo-Shaeer, and W. Ketterle, preprint cond-mat/0008423.

33. B. Jackson, J. F. McCann, and C. S. Adams, Phys. Rev. A 61, 051603(R) (2000).

34. T. Frisch, Y. Pomeau, and S. Rica, Phys. Rev. Lett. 69, 1644 (1992).

35. B. Jackson, J. F. McCann, and C. S. Adams, Phys. Rev. Lett. 80, 3903 (1998).

36. T. Winiecki, J. F. McCann, and C. S. Adams, Phys. Rev. Lett. 82, 5186 (1999).

37. L. V. Hau, S. E. Harris, Z. Dutton, and C. H. Behroozi, Nature 397, 594 (1999).

38. A. Görlitz, A. P. Chikkatur, and W. Ketterle, preprint, cond-mat/0008067.

39. W. Ketterle and S. Inouye, preprint, cond-mat/0008232. 